\title{
Congenital adrenal hyperplasia due to cytochrome P450 oxidoreductase deficiency
}

INSERM

\section{Source}

INSERM. (1999). Orphanet: an online rare disease and orphan drug data base. Congenital adrenal hyperplasia due to cytochrome P450 oxidoreductase deficiency. ORPHA:95699

Congenital adrenal hyperplasia due to cytochrome P450 oxidoreductase deficiency is a unique form of congenital adrenal hyperplasia ( $\mathrm{CAH}$; see this term) characterized by glucocorticoid deficiency, severe sexual ambiguity in both sexes and skeletal (especially craniofacial) malformations. 